\title{
Weight-Based Combination Therapy with Peginterferon $\alpha$-2b and Ribavirin for Naïve, Relapser and Non-Responder Patients with Chronic Hepatitis C
}

\author{
Fernando Lopes Gonçales Jr. ${ }^{1}$, Aline Vigani', \\ Neiva Gonçales ${ }^{1}$, Antonio Alci Barone ${ }^{2}$, Evaldo Araújo ${ }^{2}$, \\ Roberto Focaccia ${ }^{3}$, Umbeliana Oliveira ${ }^{3}$, \\ Henrique Sérgio Morais Coelho ${ }^{4}$, Jacqueline Paixao ${ }^{4}$, \\ Renata Perez ${ }^{5}$, Cirley Lobato ${ }^{6}$, Judith Weirich ${ }^{6}$, \\ Heitor Rosa ${ }^{7}$, Andrelina Borges ${ }^{7}$, Ricardo Vila $^{8}$, \\ Maria Lúcia Corrêa-Giannella ${ }^{9}$ and Maria Lúcia Gomes Ferraz ${ }^{5}$
}

\begin{abstract}
${ }^{1}$ Faculty of Medical Sciences of Campinas, Campinas, SP; ${ }^{2}$ School of Medicine of São Paulo University, São Paulo, SP; ${ }^{3}$ Emilio Ribas Institute, São Paulo, SP; ${ }^{4}$ Federal University of Rio de Janeiro, Rio de Janeiro, RJ; ${ }^{5}$ Federal University of São Paulo, São Paulo, SP; ${ }^{6}$ Secretary of Health of Acre; Rio Branco, AC; ${ }^{7}$ Federal University of Goiás, Goiânia, GO; ${ }^{8}$ Schering-Plough Brazil, São Paulo, SP; ${ }^{9}$ Laboratory of Medical Investigations (LIM-25) of Medical School of São Paulo University, São Paulo, SP; Brazil
\end{abstract}

\begin{abstract}
Combination therapy with pegylated interferon and ribavirin is considered the new standard therapy for naïve patients with chronic hepatitis $\mathrm{C}$. We evaluated the efficacy and safety of treatment with weight-based peginterferon $\alpha-2 b(1.5 \mathrm{mg} / \mathrm{kg}$ per week) plus ribavirin $(800-1,200 \mathrm{mg} / \mathrm{day})$ for 48 weeks in naïve, relapser and non-responder (to previous treatment with interferon plus ribavirin) patients with chronic hepatitis C. Sixty-seven naïve, 26 relapser and 40 non-responder patients were enrolled. The overall sustained virological response (SVR) for the intention-to-treat population was $54 \%$ for naïve, $62 \%$ for relapser and $38 \%$ for non-responder patients. In the naïve subgroup, SVR was significantly higher in patients with the non-1 genotype $(67 \%)$ compared to those with genotype 1 $(45 \%)$. In relapsers and non-responders, SVR was, respectively, $69 \%$ and $24 \%$ in patients with genotype 1 and $43 \%$ and $73 \%$ in those with genotype non-1. There were no significant differences in SVR rates among the three body weight ranges $(<65 \mathrm{~kg}, 65-85 \mathrm{~kg}$ and $>85 \mathrm{~kg}$ ) in any of the subgroups. Early virological response (EVR) was reached by $78 \%, 81 \%$ and $58 \%$ of naïve, relapser and non-responder patients, respectively, and among those with EVR, 63\%, $67 \%$ and $61 \%$, respectively, subsequently achieved SVR. All of the non-responder patients who did not have EVR reached SVR. Treatment was discontinued in $13 \%$ of the patients, due to loss to follow-up, hematological abnormalities or depression.
\end{abstract}

Key Words: Hepatitis C, interferon $\alpha-2 b$, therapy.

Chronic hepatitis $\mathrm{C}$ promotes a slow but progressive deterioration of liver function in up to $70 \%$ of affected individuals [1]. Treatment for hepatitis $\mathrm{C}$ has evolved from the use of interferon-a monotherapy to combination therapy with interferon plus ribavirin [2-5], and more recently to combination therapy with pegylated interferon $\alpha-2 b$ or $\alpha-2 a$ once a week. These regimens have given a significantly higher sustained-virological-response (SVR) rate than combination therapy with interferon and ribavirin in the treatment of naïve patients (54\%-56\% vs. 44\%-47\%) [6,7]; they are considered the new standard therapy for this group of patients [8]. Despite similar SVR rates observed with the two pegylated interferons, the use of these two drugs differs significantly, since peginterferon $\alpha$-2a is given in fixed doses, while peginterferon $\alpha-2 b$ is dosed according to the patient's weight, based on

Received on 07 June 2006; revised 16 September 2006.

Address for correspondence: Dr. Maria Lúcia Gomes Ferraz. Rua Machado Bittencourt 413, apto 81, Zip code: 04044-001, São Paulo - SP, Brazil. This study was sponsored by Schering-Plough. The authors declare conflict of interest.

The Brazilian Journal of Infectious Diseases 2006;10(5):311-316. (C) 2006 by The Brazilian Journal of Infectious Diseases and Contexto Publishing. All rights reserved. previous findings that response rates to interferon $\alpha-2 b$ monotherapy were strongly influenced by body weight [9]. We evaluated the efficacy and safety of weight-based peginterferon $\alpha-2 b$ and ribavirin treatment of Brazilian patients with chronic hepatitis $\mathrm{C}$. Since the efficacy of peginterferon in the retreatment of patients who have not achieved SVR with interferon plus ribavirin is not well established, we included patients that had been non-responders or who had relapsed in response to previous interferon plus ribavirin therapy, along with naïve patients.

\section{Material and Methods}

\section{Study design}

This was an open, prospective, national, multicenter study, approved by the institutional ethics committee of all seven participating centers; all patients provided signed informed consent. Patients who met the inclusion criteria received a combination of peginterferon $\alpha$ - $2 b$ plus ribavirin for 48 weeks. Peginterferon $\alpha-2 b$ (PegIntron, Schering Plough, Kenilworth, NJ, USA) was used at a dose of $1.5 \mathrm{mg} / \mathrm{kg}$ per week subcutaneously in a single injection. Ribavirin (Rebetol, Schering Plough, Kenilworth, NJ, USA) was given orally twice a day at a total daily dose of $800 \mathrm{mg}$ (naïve patients: body 
weight $<65 \mathrm{~kg}$ ), 1,000 mg (naïve patients: body weight $65-85$ $\mathrm{kg}$; relapsers and non-responders: $<75 \mathrm{~kg}$ ) or $1,200 \mathrm{mg}$ (naïve patients: body weight $>85 \mathrm{~kg}$; relapsers and non-responders $>75 \mathrm{~kg}$ ).

Stepwise reductions in peginterferon $\alpha$ - $2 \mathrm{~b}$ dosages to 1.0 $\mathrm{mg} / \mathrm{kg}$ per week were made whenever platelets fell below $80,000 / \mathrm{mm}^{3}$ or granulocytes fell below 750 cells $/ \mathrm{mm}^{3}$. Reductions in ribavirin dosages to half of initial doses were made whenever hemoglobin levels fell below $10 \mathrm{mg} / \mathrm{dL}$. Full doses were restarted after normalization of the event.

All patients were evaluated for safety and tolerance at the end of weeks 2, 4, 8, 12, and every 6 weeks during treatment. Biochemical (ALT, AST, glycemia, creatinine, bilirubin, GGT, alkaline phosphatase, total protein, albumin) and hematology exams (complete blood count, platelet count) tests were made at every visit. Thyroid function (TSH and free T4) was assessed at baseline and at weeks 8 and 24. Serum HCV-RNA levels were quantitatively assessed by RT- PCR assay (Cobas Amplicor HCV test, version 2.0; lower limit of detection, 100 copies [50 IU] per $\mathrm{mm}^{3}$ ) at baseline and at week 12, while qualitative RT-PCR was done at week 48 (end of treatment) and at follow-up (24 weeks after the end of treatment).

EVR was defined as either undetectable serum HCV-RNA or a S 2-log decline in serum HCV-RNA, based on quantitative RT-PCR assays, by week 12 of treatment. SVR was defined as absence of detectable HCV-RNA at the end of follow-up (24 weeks after the end of treatment) by qualitative RT-PCR assay.

\section{Patient selection}

Eligible subjects were previously untreated (naïve) patients (15-65 years old), previously treated with interferon and ribavirin who presented detectable HCV RNA measured during or following treatment after a period of undetectable HCV RNA (relapsing patients) and those previously treated with interferon and ribavirin who continued viremic throughout treatment and follow-up (non-responder patients). All patients had detectable HCV-RNA in the serum, abnormal serum ALT concentrations for at least six months before the beginning of the protocol, and a liver biopsy specimen collected within 18 months of entry in the study, with histopathological confirmation of chronic hepatitis C. Histological activity of 81 out of 133 cases $(61 \%)$ was revised by a center pathologist using the METAVIR Scoring System: 39 out of 67 naïve patients (58\%), 23 out of 40 non-responders (58\%) and 19 out of $26(73 \%)$ relapsers. Fibrosis of each liver biopsy specimen was staged on a scale of 0 to 4 ( 0 , no fibrosis; 1 , portal fibrosis without septa formation; 2 , few septa; 3 , numerous septa without cirrhosis; 4 , cirrhosis). The activity, measured as the intensity of necroinflammatory lesions, was scored as follows: A0, no histological activity; A1, mild activity; A2, moderate activity; and A3, severe activity) [10,11].

Patients were excluded from the study if they had hemoglobin values $<12 \mathrm{~g} / \mathrm{dL}$, neutrophils $<1,500 / \mathrm{mm}^{3}$, platelets $<100,000 / \mathrm{mm}^{3}$, human immunodeficiency virus (HIV) infection, coinfection with hepatitis B, decompensated liver disease, seizure disorders, previous organ transplantation, significant cardiovascular dysfunction, chronic pulmonary disease, poorly controlled diabetes, other cause of liver disease, preexisting psychiatric disease, hemoglobinopathies, hemophilia, clinically significant retinal abnormalities or immunologicallymediated disease. Laboratory values for serum bilirubin, prothrombin time, albumin, glycemia, creatinine and alphafetoprotein had to be within the normal range. All patients had an ultrasonography evaluation at least three months before entry in the study with no evidence of nodules or hepatocarcinoma. Patients were also excluded if they were consuming alcohol or using intravenous or inhaled drugs on a regular basis, were pregnant or nursing. Non-responders and relapsers must have finished previous interferon plus ribavirin treatment at least six months previously.

\section{Endpoints}

The primary endpoint for this study was SVR, defined as undetectable HCV-RNA in the serum 24 weeks after the end of treatment. EVR and safety were secondary endpoints. Results were recorded for the intention-to-treatment (ITT) population (all patients who received at least one dose of the study medication) as well as for the on-treatment population.

\section{$\underline{\text { Statistical analysis }}$}

Contingency tables were used for comparing the distribution of SVR across factor levels (genotype, viral load, body weight and degree of fibrosis). The chi-square test was employed for evaluating statistical differences with $\mathrm{P}<0.05$.

\section{Results}

\section{Baseline characteristics}

From March 2001 to December 2002, 133 patients were enrolled and received at least one dose of the medication; they were comprised of 67 naïve, 26 relapser and 40 nonresponder patients. Baseline host and viral characteristics are shown in Table 1. Most of the patients were male (67\%), with a mean age of $44 \pm 0.85$ years and a mean body mass index of $26.5 \pm 0.34 \mathrm{~kg} / \mathrm{m}^{2}$. HCV genotype 1 was the most frequent in this series $(66 \%)$. and HCV-RNA was > 800,000 copies/mL in $39 \%$ of the patients.

\section{Efficacy of therapy}

Of the 133 included patients, 116 completed therapy (59 naïve, 23 relapser and 34 non-responder patients) and were considered in the on-treatment analysis. SVR results for the intention-to-treat population and the on-treatment population are shown in Table 2. The overall SVR was 54\% for naïve patients, $62 \%$ for relapsers and $38 \%$ for non-responders in the intention-to-treat population and $61 \%, 70 \%$ and $44 \%$, respectively, for the on-treatment population. In the naïve subgroup, SVR was significantly higher in patients with 
Table 1. Baseline characteristics of chronic hepatitis $\mathrm{C}$ patients

\begin{tabular}{|c|c|c|c|}
\hline & Naïve (n=67) & Relapser $(n=26)$ & Non-responder $(n=40)$ \\
\hline Sex (\% Male) & $64 \%$ & $62 \%$ & $75 \%$ \\
\hline Age (years, mean \pm SD [Range]) & $43.3 \pm 0.96(24.0-61.0)$ & $45.5 \pm 2.64(20.0-68.0)$ & $44.3 \pm 1.61(15.0-65.0)$ \\
\hline Body weight (kg, mean \pm SD [Range]) & $77.0 \pm 1.69(52.0-112.0)$ & $75.0 \pm 2.92(45.2-102.0)$ & $75.3 \pm 1.98(46.8-101.0)$ \\
\hline BMI $\left(\mathrm{kg} / \mathrm{m}^{2}\right.$, mean $\pm \mathrm{SD}$ [Range] $)$ & $26.7 \pm 0.47(20.6-37.6)$ & $25.7 \pm 0.79(18.8-33.1)$ & $26.6 \pm 0.63(20.0-36.0)$ \\
\hline Genotype 1 & $60 \%$ & $73 \%$ & $73 \%$ \\
\hline High viral load (> 800,000 copies/mL) & $40 \%$ & $31 \%$ & $43 \%$ \\
\hline Degree of fibrosis* & (Available in 39 cases) & (Available in 19 cases) & (Available in 23 cases) \\
\hline $\mathrm{F} 0-\mathrm{F} 2$ & $64 \%$ & $74 \%$ & $74 \%$ \\
\hline $\mathrm{F} 3$ & $26 \%$ & $21 \%$ & $13 \%$ \\
\hline $\mathrm{F} 4$ & $10 \%$ & $5 \%$ & $13 \%$ \\
\hline HCV-RNA (copies/mL, mean \pm SEM) & $910,425 \pm 157,820$ & $660,833 \pm 114,108$ & $1,018,131 \pm 197,534$ \\
\hline $\mathrm{ALT}($ mean $\pm \mathrm{SEM})$ & $146.2 \pm 13.3$ & $83.3 \pm 6.5$ & $130.6 \pm 15.7$ \\
\hline $\begin{array}{l}\text { Normal limits: } \\
\text { Female: } 10-36 \mathrm{U} / \mathrm{L} \\
\text { Male: } 10-44 \mathrm{U} / \mathrm{L}\end{array}$ & & & \\
\hline
\end{tabular}

SD: Standard deviation; BMI: Body mass index; SEM: Standard error of the media. * Available in 81 out of 133 cases $(61 \%)$.

Table 2. Treatment response by week 12 (early virological response [EVR]), end of treatment and end of follow-up (sustained virological response [SVR]): intention-to-treat (ITT) and on-treatment analysis of chronic hepatitis C patients

\begin{tabular}{|c|c|c|c|c|c|c|}
\hline \multirow[t]{2}{*}{ Treatment response } & \multicolumn{2}{|c|}{ Naïve } & \multicolumn{2}{|c|}{ Relapsers } & \multicolumn{2}{|c|}{ Non-responders } \\
\hline & ITT & On-treatment & ITT & On-treatment & ITT & On-treatment \\
\hline \multicolumn{7}{|l|}{ Overall } \\
\hline 12 weeks (EVR) & $78 \%(52 / 67)^{\bullet}$ & $88 \%(52 / 59)$ & $81 \%(21 / 26)^{\mathbb{I}}$ & $91 \%(21 / 23)$ & $58 \%(23 / 40)^{£}$ & $68 \%(23 / 34)$ \\
\hline End of treatment & $67 \%(45 / 67)$ & $76 \%(45 / 59)$ & $73 \%(19 / 26)$ & $83 \%(19 / 23)$ & $45 \%(18 / 40)$ & $53 \%(18 / 34)$ \\
\hline End of follow-up (SVR) & $54 \%(36 / 67)$ & $61 \%(36 / 59)$ & $62 \%(16 / 26)$ & $70 \%(16 / 23)$ & $38 \%(15 / 40)$ & $44 \%(15 / 34)$ \\
\hline \multicolumn{7}{|l|}{ SVR by genotype ${ }^{\#}$} \\
\hline 1 & $45 \%(18 / 40)$ & $49 \%(18 / 37)$ & $69 \%(13 / 19)$ & $72 \%(13 / 18)$ & $24 \%(7 / 29)$ & $28 \%(7 / 25)$ \\
\hline Non-1 & $67 \%(18 / 27)$ & $82 \%(18 / 22)$ & $43 \%(3 / 07)$ & $60 \%(3 / 5)$ & $73 \%(8 / 11)$ & $89 \%(8 / 9)$ \\
\hline \multicolumn{7}{|l|}{ SVR by baseline $\mathrm{HCV}^{\S}$} \\
\hline$<800,000$ copies $/ \mathrm{mL}$ & $58 \%(23 / 40)$ & $66 \%(23 / 35)$ & $56 \%(10 / 18)$ & $63 \%(10 / 16)$ & $40 \%(9 / 23)$ & $43 \%(9 / 21)$ \\
\hline$>800,000$ copies $/ \mathrm{mL}$ & $48 \%(13 / 27)$ & $54 \%(13 / 24)$ & $75 \%(6 / 8)$ & $88 \%(6 / 7)$ & $35 \%(6 / 17)$ & $46 \%(6 / 13)$ \\
\hline \multicolumn{7}{|l|}{ SVR by body weight * } \\
\hline$<65 \mathrm{~kg}$ & $57 \%(8 / 14)$ & $67 \%(8 / 12)$ & $78 \%(7 / 9)$ & $78 \%(7 / 9)$ & $25 \%(2 / 8)$ & $29 \%(2 / 7)$ \\
\hline $65-85 \mathrm{~kg}$ & $49 \%(17 / 35)$ & $55 \%(17 / 31)$ & $71 \%(5 / 7)$ & $71 \%(5 / 7)$ & $42 \%(10 / 24)$ & $48 \%(10 / 21)$ \\
\hline$>85 \mathrm{~kg}$ & $61 \%(11 / 18)$ & $69 \%(11 / 16)$ & $40 \%(4 / 10)$ & $57 \%(4 / 7)$ & $38 \%(3 / 8)$ & $50 \%(3 / 6)$ \\
\hline \multicolumn{7}{|l|}{ SVR by degree of fibrosis \& } \\
\hline $\mathrm{F} 0-\mathrm{F} 2$ & $60 \%(15 / 25)$ & $68 \%(15 / 22)$ & $57 \%(8 / 14)$ & $67 \%(8 / 12)$ & $38 \%(6 / 16)$ & $40 \%(6 / 15)$ \\
\hline F3-F4 & $29 \%(4 / 14)$ & $40 \%(4 / 10)$ & $80 \%(4 / 5)$ & $80 \%(4 / 5)$ & $43 \%(3 / 7)$ & $75 \%(3 / 4)$ \\
\hline
\end{tabular}

- Two patients did not have an HCV-RNA exam at week 12 and one patient dropped out at week 2.

"One patient dropped out at week 2 .

${ }^{£}$ One patient did not have an HCV-RNA exam at week 12 and three patients dropped out at week 8 .

\# SVR rate was significantly higher in genotype non-1 compared to genotype 1 only in the naïve subgroup.

$\S$ There were no significant differences in SVR rates between high and low viral load in each subgroup.

* There were no significant differences in SVR rates among the three body weight ranges in each subgroup.

\& There were no significant differences in SVR rates between F0-F2 and F3-F4 in each subgroup. 
genotype non-1 (67\%) compared to patients with genotype 1 $(45 \%)(\mathrm{P}<0.05)$. In relapsers and non-responders, SVR was, respectively, $69 \%$ and $24 \%$ in patients with genotype 1 and $43 \%$ and $73 \%$ in those with genotype non- 1 .

Among naïve, relapser and non-responder patients with high baseline viral load, $48 \%, 75 \%$ and $35 \%$, respectively, reached SVR, while in patients with low baseline viral load, $58 \%, 56 \%$ and $40 \%$, respectively, achieved SVR. As shown in Table 2, there were no significant differences in SVR rates among the three body weight ranges $(<65 \mathrm{~kg}, 65-85 \mathrm{~kg}$ and $>$ $85 \mathrm{~kg}$ ) in any of the subgroups. Among naïve, relapser and non-responder patients with an F0-F2 fibrosis score, $60 \%$, $57 \%$ and $38 \%$, respectively, had SVR, while in patients with an F3-F4 fibrosis score, 29\%, $80 \%$ and 43\%, respectively, achieved SVR. The biochemical (alanine aminotransferase) response rates at the end of treatment and at the end of follow up were, respectively, $79 \%$ and $74 \%$ for naïve patients, $90 \%$ and $86 \%$ for relapsers and $66 \%$ and $45 \%$ for non-responders. Nearly all naïve patients and relapsers who achieved SVR also had normal ALT values at the end of follow up: $97 \%$ and $93 \%$, respectively, while normal ALT values were observed in $80 \%$ of non-responders who reached SVR.

EVR was reached by $78 \%, 81 \%$ and $58 \%$ of naïve, relapser and non-responder patients, respectively, and among those with EVR, 63\%, 67\% and 61\% subsequently achieved SVR. Among the 12 naïve patients who did not have EVR, 10 patients completed therapy and four reached SVR. Among the four relapser patients who did not present EVR, three patients completed therapy and two achieved SVR. Among the 13 non-responder patients who did not have EVR, 12 patients completed therapy and none $(0 \%)$ reached SVR.

\section{Safety}

Treatment was discontinued in 17 patients (13\%) due to abnormalities in the laboratory tests or other adverse events: $12 \%$ of the naïve patients, $11 \%$ of relapsers and $15 \%$ of nonresponders. The reasons for treatment discontinuation were loss of follow-up (7/17) depression (6/17), neutropenia (2/17), anemia (1/17) and low platelet counts (1/17). The percentage of patients reporting the most common adverse events (fever, myalgia, headache, fatigue, nausea, rigors and decreased appetite) decreased with time. Alopecia was more frequently reported after treatment week 18 . The number of patients reporting irritability, insomnia and depression was low and remained constant throughout the entire treatment period.

Hemoglobin concentrations fell after week 2, stabilized after week 12 and returned to baseline values during followup. Granulocyte counts decreased after week 2 , stabilized after week 8 and returned to baseline values during follow-up. Platelet counts reached the lowest values on week 8; then they gradually increased and normalized during follow-up (Figure 1). The temporal pattern of decrease of hematological variables did not differ among the subgroups of naïve, relapser and non-responder patients. Decreases in hematological variables, requiring reduction of ribavirin and/or peginterferon $\alpha-2 b$ doses, were observed as follows for naïve, relapsers and non-responder patients, respectively: hemoglobin below 10 $\mathrm{mg} / \mathrm{dL}, 10 \%, 11.5 \%$ and $22 \%$; granulocytes below 750 cells/ $\mathrm{mL}, 22 \%, 19 \%$ and $20 \%$, and platelets below 80,000 cells $/ \mathrm{mL}$, $4 \%, 4 \%$ and $17 \%$.

Body weight decreased progressively throughout the treatment period and stabilized after week 30 (Figure 1). Mean body weight loss at the end of therapy was $8 \%$. A significant increase in ALT concentration (greater than five times baseline) was observed in only one patient (from the relapser group) during the study.

\section{Discussion}

In this study carried out in a Brazilian population, with the administration of weight-based peginterferon $\alpha-2 b$ at a dose of $1.5 \mathrm{mg} / \mathrm{kg}$ per week plus ribavirin for 48 weeks, the success rates observed for naïve patients were similar to those observed by Manns et al. [6], with an overall SVR rate of 54\% in the intention-to-treat population and $61 \%$ in the ontreatment population. The SVR rates in the population infected with genotype 1 (45\% in the intention-to-treat analysis and $49 \%$ in the on-treatment analysis) were also similar to those described by Manns et al. Liver histology was reviewed by the same pathologist in $58 \%$ of the naive patients; the evaluation of this subgroup showed a SVR rate of $60 \%$ in the intention-to-treat analysis and $68 \%$ in the on-treatment analysis, in patients with fibrosis grade 0 to 2 , and a SVR rate of $29 \%$ in the intention-to-treat analysis and $40 \%$ in the ontreatment analysis in patients with fibrosis grades 3 and 4 . The lack of significant differences in SVR rates between patients with minimal versus advanced fibrosis degree as well as between patients with low versus high viral load could be due to the small sample size.

The SVR results in relapsers who had previously received interferon plus ribavirin therapy confirmed the conclusions of a previous study that stated that these patients are an ideal subgroup of patients for retreatment [12], since an overall SVR rate of $62 \%$ in the intention-to-treat analysis and $70 \%$ in the on-treatment analysis was observed. These results were similar to those reported by Portal et al. in a study of 46 relapsers who received peginterferon $\alpha-2 b$ at a dose of 0.7 $\mathrm{mg} / \mathrm{kg}$ per week plus ribavirin (800-1,000 mg/day) for 48 weeks or peginterferon $\alpha-2 b$ at a dose of $1.5 \mathrm{mg} / \mathrm{kg}$ per week for eight weeks, followed by peginterferon $\alpha-2 b$ at a dose of $0.7 \mathrm{mg} / \mathrm{kg}$ for 40 weeks plus ribavirin (800-1,000 mg/day) for 48 weeks. The SVR rates did not differ between the two treatment groups, with an overall SVR of 68\% [13]. High SVR rates were observed in patients with genotype 1 both in our sample $(69 \%$ in the intention-to-treat population) and in the population studied by Portal et al. (69\% and 73\%). In the EPIC 3 trial, Poynard et al. reported an overall SVR of $39 \%$ in relapsers receiving peginterferon $\alpha-2 b$ at a dose of $1.5 \mathrm{mg} / \mathrm{kg}$ per week plus ribavirin (800-1,400 mg/day) for 48 weeks. Among patients 
with genotype 1, only $29 \%$ reached SVR [14]. Differences in the characteristics of these populations might explain the differences found in the SVR rates between the studies. We found that $69 \%$ of the relapsers in our sample had a low viral load, which could partially explain the high SVR rates.

SVR rates attained by non-responders $(38 \%$ in the intention-to-treat analysis and $44 \%$ in the on-treatment analysis), although much lower than those reported for the group of relapsers, were higher than those observed in other studies that included non-responders to prior interferon plus ribavirin therapy. In the EPIC3 trial, an overall SVR of $15 \%$ was observed in non-responders receiving peginterferon $\alpha-2 b$ at a dose of $1.5 \mathrm{mg} / \mathrm{kg}$ per week plus ribavirin (800-1,400 mg/day) for 48 weeks [14]. Shiffman et al. treated 385 previous nonresponders to interferon plus ribavirin with peginterferon $\alpha$ $2 \mathrm{a}$ at a dose of $180 \mathrm{mg}$ per week, along with ribavirin $(1,000-$ $1,200 \mathrm{mg} / \mathrm{d}$ ), and observed an overall SVR rate of $12 \%$ [15]. When compared to our population, the population studied by Shiffman had a higher percentage of genotype 1 carriers ( $89 \mathrm{vs.} .73 \%$ ) and of patients with high viral load (75 vs. 43\%), in addition to a higher mean body weight $(89 v s .75 \mathrm{~kg})$, which could in part explain the large difference in the SVR rate observed in these two studies. The $17 \%$ rate of relapse observed in the non-responders in our study was similar to the rate observed for the naïve subgroup (20\%) and lower than that reported by Shiffman $(50 \%)$, which the author suggests to be the result of the high degree of ribavirin dose reduction (nearly 40\%) [15]. Whether the low rate of ribavirin dose reduction in the non-responder patients in our study (22\%) contributed to the low rate of relapse remains to be investigated. Despite the small sample size, we found a high SVR rate in non-responder patients with genotypes 2 and 3 in our study ( $73 \%$ in the intention-to-treat analysis).
Analyses of the results of clinical trials with interferon a$2 \mathrm{~b}$ monotherapy have shown that SVR rates are strongly influenced by body weight, since greater body weights were associated with lower SVR [9]. The impact of body weight on SVR was also reported by a peginterferon $\alpha$-2a monotherapy study [16] and by a peginterferon $\alpha$-2a plus ribavirin study, in which a body weight $\leq 75 \mathrm{~kg}$ was shown to independently and significantly increase the odds of achieving SVR [7]. With the use of weight-based peginterferon $\alpha-2 b$ plus ribavirin in our study, no significant differences were observed in SVR rates for the three different body weight ranges, i.e., $<65 \mathrm{~kg}$, $65-85 \mathrm{~kg}$ and $>85 \mathrm{~kg}$ in any of the subgroups. In addition to allowing patients with higher body weight to reach SVR rates similar to those achieved by patients with lower body weight, the administration of weight-based peginterferon $\alpha-2 b$ allows dose adjustment, as body weight decreases during the treatment, which could have a direct impact on the frequency of adverse events and, consequently, on the compliance of patients with significant weight loss secondary to treatment.

PCR analysis at week 12 showed that the percentage of patients with EVR was similar for naïve patients (78\%) and relapsers $(81 \%)$, as was as the percentage of patients with EVR who achieved SVR (63\% e 67\%, respectively). Differently from the report by Davis et al. for a phase III peginterferon $\alpha$ $2 \mathrm{~b}$ plus ribavirin clinical trial, in which any patient who did not reach EVR achieved SVR [17], four out of 10 naïve patients and two out of three relapsers who did not have EVR achieved SVR. In spite of the lower EVR rate observed in non-responders $(58 \%), 61 \%$ of these patients reached SVR. All of the nonresponders who did not have EVR reached SVR, which corroborates previous findings that the performance of quantitative PCR after 12 weeks of therapy with pegylated interferon is also valuable for maintenance of therapy in non-

Figure 1. Mean hemoglobin, granulocyte and platelet counts, and mean body weight loss during and after treatment in the three subgroups.
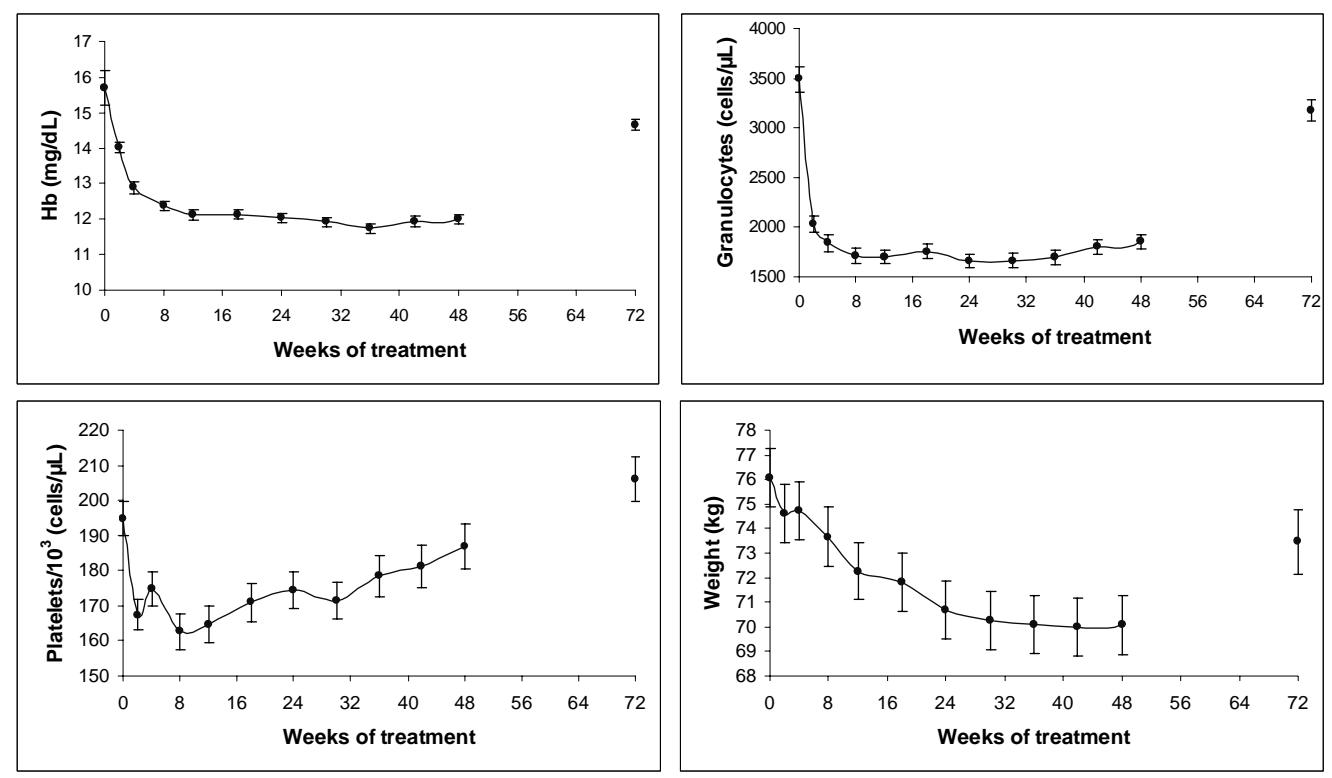
responders receiving combination therapy with peginterferon plus ribavirin [15].

The rate of the most common adverse events (flu-like symptoms) decreased over time, whereas the rate of depression and irritability remained relatively steady during the 48 weeks of treatment. The adverse event that promoted the highest discontinuation rate was depression. Decreases in hemoglobin and platelet count values requiring ribavirin and peginterferon $\mathrm{a}-2 \mathrm{~b}$ dose reductions were similar to those previously reported for naïve patients [6] in the subgroups of naïve and relapser patients, whereas in the subgroup of non-responders, decreases in hemoglobin and platelet count values requiring dose reduction were more frequent. The percentage of patients with granulocyte count decrease requiring peginterferon $\alpha$ $2 \mathrm{~b}$ dose reduction was similar in the three subgroups of patients.

In conclusion, SVR rates observed after weight-based combination therapy with peginterferon $\alpha-2 b$ plus ribavirin in naïve Brazilian patients with chronic hepatitis $\mathrm{C}$ were similar to those obtained in the pivotal multicentric phase III study [6]. Brazilian relapsers who had received prior therapy with interferon plus ribavirin had a high SVR rate and behaved similarly to the group of naïve patients in terms of the prediction value of EVR and of the frequency of abnormal hematological variables. Non-responders had a higher SVR rate than those reported in other studies that included nonresponders to prior therapy with interferon plus ribavirin; this could be at least partly explained by differences in the baseline characteristics of the study populations; however, these results need confirmation in a larger-scale study. In agreement with a previous report [15], PCR carried out on week 12 seems to be important in non-responders, since the chances of reaching SVR in patients who did not have EVR are minimal. This is an important finding, not only because of the savings it might entail, but also to decrease the exposure of patients, with fewer chances of adverse events, especially if other studies confirm the findings that this group of patients is more prone to greater hemoglobin and platelet count decreases than the group consisting of naïve and relapser patients. Further SVR analysis stratified by body weight might be performed to check our conclusion that administration of weight-based peginterferon $\alpha-2 b$ and ribavirin overcomes the impact of body weight on SVR rates.

\section{References}

1. Alter M.J., Margolis H.S., Krawczynski K., et al. The natural history of community-acquired hepatitis $\mathrm{C}$ in the United States. N Engl J Med 1992;327:1899-905.

2. Davis G.L., Balart L.A., Schiff E.R., et al. Treatment of chronic hepatitis $\mathrm{C}$ with recombinant interferon alfa. N Eng J Med 1989; $321: 1501-6$.
3. Di Bisceglie A.M., Martin P., Kassianides C., et al. Recombinant interferon alfa therapy for chronic hepatitis C. N Eng J Med 1989;321:1506-10.

4. McHutchison J.G., Gordon S.C., Schiff E.R., et al. Interferon alfa $2 \mathrm{~b}$ alone or in combination with ribavirin as initial treatment for chronic hepatitis C. N Engl J Med 1998;339: 1485-92.

5. Poynard T., Marcellin P., Lee S.L., et al. Randomised trial of interferon alpha $2 \mathrm{~b}$ plus ribavirin for 48 weeks or for 24 weeks versus interferon alpha $2 \mathrm{~b}$ plus placebo for 48 weeks for treatment of chronic infection with hepatitis $\mathrm{C}$ virus. Lancet 1998;352:1426- 32.

6. Manns M.P., McHutchison J.G., Gordon S.C., et al. PegInterferon alfa-2b plus ribavirin compared with interferon alfa-2b plus ribavirin for initial treatment of chronic hepatitis C: a randomised trial. Lancet 2001;358:958-65.

7. Fried M.W., Shiffman M.L., et al. Peginterferon alfa-2a plus ribavirin for chronic hepatitis $\mathrm{C}$ virus infection. $\mathrm{N}$ Engl $\mathrm{J}$ Med 2002;347:975-82.

8. National Institutes of Health Consensus Development Conference Statement: Management of hepatitis C. Gastroenterology 2002;123:2082-99.

9. McHutchison J.G., Poynard T., Salpetriere P., et al. Patient body weight and response to interferon alfa $2 \mathrm{~b}$ monotherapy [abstract no. 998]. In: 52 $2^{\text {nd }}$ Annual Meeting of the American Association for the Study of Liver Diseases (AASLD) (Dallas) Texas: 2001.

10. The French METAVIR Cooperative Study Group. Intraobserver and interobserver variations in liver biopsy interpretation in patients with chronic hepatitis C. Hepatology 1994;20(1 Pt 1):15-20.

11. Bedossa P., Poynard T. An algorithm for the grading of activity in chronic hepatitis C. The METAVIR Cooperative Study Group. Hepatology 1996;24:289-93.

12. Shiffman M.L. Management of Patients With Chronic Hepatitis C Virus Infection and Previous Nonresponse. Rev Gastroenterol Disord 2004;4(suppl 1):S22-S30.

13. Portal I., Botta-Fridlund D., Bourliere M., et al. Treatment with pegylated-interferon alpha-2B (PEG-interferon) + ribavirin in relapsers to standard interferon + ribavirin in chronic hepatitis $\mathrm{C}$ : efficacy and safety results from a randomized multicentric french study. Hepatology 2002;36:359A.

14. Poynard T., Schiff E., Terg R., et al. Sustained virologic response (SVR) in the EPIC3 trial: week twelve virology predicts SVR in previous interferon/ribavirin treatment failures receiving Peg-Intron/Rebetol (PR) weight based dosing (WBD). Journal of Hepatology 2005;42(suppl 2):40.

15. Shiffman M.L., Di Bisceglie A.M., Lindsay K.L., et al. Peginterferon alfa-2a and ribavirin in patients with chronic hepatitis $\mathrm{C}$ who have failed prior treatment. Gastroenterology 2004; $126: 1015-23$.

16. Zeuzem S., Feinman V., Rasenack J., et al. Peginterferon alfa-2a in patients with chronic hepatitis C. N Engl J Med 2000;343:1666-72.

17. Davis G.L., Wong J.B., McHutchison J.G., et al. Early virologic response to treatment with peginterferon alfa- $2 \mathrm{~b}$ plus ribavirin in patients with chronic hepatitis C. Hepatology 2003;38:645-52. 